\title{
EDITORIAL
}

\section{"I am not depressed. I just don't like myself": stigma and (lack of) depression in a subset of patients with Parkinson's disease}

\author{
Raja Mehanna (iD \\ UT MOVE, Department of Neurology, University of Texas Health Science at Houston, Houston, TX, USA.
}

Defined as a feeling of disapproval that people have about particular illnesses or ways of behaving, stigma is no stranger to patients suffering from Parkinson's disease (PD). Indeed, the majority of PD patients report it and attempt to mask their symptoms in social circumstances, ${ }^{1}$ while healthy people acknowledge that they would want to hide their symptoms of PD if they were ever affected by it. ${ }^{2}$ However, the impact of self-stigma goes beyond a subjective feeling. It can lead to a reluctance to seek medical assistance or to ask for adjustments at work, which in turn decreases the chances of staying employed. Stigma can, by definition, impact the social life and thus social support of PD patients, leading to isolation. It can ultimately increase the risk of depression and even suicide. $^{3}$ In a hierarchical multiple regression study, Ma et al. $^{4}$ reported that stigma was a key determinant of quality of life in patients with PD, with patients with higher stigma having more depressive symptoms, more severe $\mathrm{PD}$, and more difficulty with activities of daily living.

In the current issue of the Brazilian Journal of Psychiatry, da Silva et al. ${ }^{5}$ focus on the specific subset of PD patients who are candidate for deep brain stimulation (DBS). Because these patients are usually more advanced in their disease progression, the authors hypothesized that depressive symptoms would be associated with increased self-stigma, regardless of the severity of their motor symptoms. In their cross-sectional study, they reviewed a sample of 54 PD patients who were DBS candidates and investigated clinical and sociodemographic variables associated with stigma. The independent variables were age, disease duration, motor symptoms assessed by the motor section of Movement Disorder Society Unified Parkinson Disease Rating Scale (MDS-UPDRS III), depressive symptoms measured by the Hospital Anxiety and Depression Scale (HADS), and the Mobility, Activities of daily living (ADL) and Emotional well-being domains of the 39-item Parkinson's Disease Questionnaire (PDQ-39). The outcome variable was the Stigma domain of the PDQ-39. The multiple linear regression reported that only the ADL domain of the PDQ 39 was independently associated with higher self-stigma, thus rejecting the study hypothesis.

Correspondence: Raja Mehanna, 6410 Fannin Street, Suite 1010, 77030, Houston, TX, USA.

E-mail: raja.mehanna@uth.tmc.edu

Submitted Jan 06 2020, accepted Jan 062020.
In this study, self-stigma thus seems related to the impact of the disease on ADL as perceived by the patient, rather than to depressive or severe motor symptoms. The message would be for the healthcare professional to center the care on the patient's perceptions and needs and not to focus solely on motor symptom control, repeating the adage of treating the patient rather than the patient's symptoms. However, a more interesting point is the lack of correlation between stigma and depressive symptoms. While stigma is frequently associated with depression, ${ }^{4}$ no correlation between the two was reported in the current study. The authors explain this by a lower level of depressive symptoms than in other studies, with at least $70 \%$ of the patients scoring below the cutoff on the depression subscale of the HADS, which is interesting considering these patients had more advanced disease as they were DBS candidates. The authors suggest that the lack of correlation between depression and self-stigma in their study could also be secondary to the more severe motor symptoms in these DBS candidate patients. This suggestion would have benefited from further explanation. Regardless of the attempts to explain these findings, larger studies on this patient population would be useful to confirm them.

Another point is that with an average age of 58.2 years and a disease duration of 14.1 years, a subset of patients likely had young-onset Parkinson's disease (YOPD). YOPD patients have a higher rate of depression than later-onset PD patients do, more stigma from their disease, as well as a higher risk of motor fluctuations and dyskinesia, which are the main indications for DBS. With the lack of correlation reported here between selfstigma and depression in DBS candidates, it would be interesting for future studies to try to replicate these findings in YOPD patients who are DBS candidates.

Finally, as the authors state, correlation does not mean causality, especially in a cross-sectional study. However, this study draws attention to perceived difficulty in ADL rather than depression or severity of motor symptoms as the main correlate to self-stigma in PD patients who are DBS candidates, suggesting screening for stigma

How to cite this article: Mehanna R. "I am not depressed. I just don't like myself": stigma and (lack of) depression in a subset of patients with Parkinson's disease. Braz J Psychiatry. 2020;42:124125. http://dx.doi.org/10.1590/1516-4446-2020-0001 
even in non-depressed patients. This could then allow environmental interventions at home and at work that might help improve the patient's self-perception, selfmanagement, and ultimately prognosis.

\section{Disclosure}

RM serves as a consultant for Global Kinetic Corporation; is on the speaker bureau for TEVA, Adamas Pharmaceuticals, Acorda Therapeuthics, and Kyowa Kirin; has received consulting fees from Amneal and Sunovion, and research grants from Lundbeck, Global Kinetics Corporation, Acorda Therapeuthics, Prilenia Therapeutics and Solstice Neurosciences.

\section{References}

1 Hermanns M. The invisible and visible stigmatization of Parkinson's disease. J Am Assoc Nurse Pract. 2013;25:563-6.

2 Moore SM, Knowles Sr. Beliefs and knowledge about Parkinson's disease. E J Appl Psychol. 2006;2:15-21.

3 Schomerus G, Evans-Lacko S, Rusch N, Mojtabai R, Angermeyer MC, Thornicroft G. Collective levels of stigma and national suicide rates in 25 European countries. Epidemiol Psychiatr Sci. 2015;24: 166-71.

$4 \mathrm{Ma} \mathrm{HI}$, Saint-Hilaire M, Thomas CA, Tickle-Degnen L. Stigma as a key determinant of health-related quality of life in Parkinson's disease. Qual Life Res. 2016;25:3037-45.

5 da Silva AG, Leal VP, da Silva PR, Freitas FC, Linhares MN, Walz R, et al. Difficulties in activities of daily living are associated with stigma in patients with Parkinson's disease who are candidates for deep brain stimulation. Braz J Psychiatry. 2020;42:190-4. 